\title{
A Preliminary Study of the Social Media Marketing under the Social Network Theory
}

\author{
Wu Mengxue \\ Shandong University at Weihai \\ Weihai, 264209 China
}

\begin{abstract}
With the development of the Internet technology, It is no doubt that it has been a profound impact on the way of people's social behavior and social communication . based on the birth of the new network tools of web 2.0, the expansion of the network social density, makes the social media arise. At present there have been some foreign social media marketing strategies which can be references. This paper Introduces the characteristics of the social media marketing, on the basis of it , analyzes the characteristics of current Chinese consumers' behavior, as well as the characteristics of the differences

between social media marketing and the traditional media , and
\end{abstract} also puts forward some marketing strategies especially to the new social media marketing enterprises .

Key words: social network; Social media marketing. The characteristics of Consumer behavior; The marketing strategy

\section{INTRODUCTION}

Social media is a kind of interactive media, namely, where the user can generate contents to be treated equally, and spread widely,which has the characteristics of authenticity, and social participation.

\section{Table 1:the characteristics of social media}

\begin{tabular}{|l|l|}
\hline sociability & $\begin{array}{l}\text { The social media has the characteristic of sociability, which refers to the " group to group" dissemination of social } \\
\text { media, the way of multi-parties involved exchange , where the users can form small groups or communities that } \\
\text { are resemble to the real-world social relationships with the topics of common interest, }\end{array}$ \\
\hline participation & $\begin{array}{l}\text { The boundaries between the media and audience socialization are very vague. The users can put forward, } \\
\text { feedback, and share information for free. This is the difference between the social media and the traditional } \\
\text { media. The traditional media is a kind of unidirectional propagation; while, the social media allows the contents to } \\
\text { be communicated between the media and the users, which reflects the participation. }\end{array}$ \\
\hline $\begin{array}{l}\text { authenticity } \\
\text { better reflection of authenticity. The social media transfers the showed relationship to the network, which } \\
\text { simulates the real world exchange. Although there is no real-name system in the social media, due to its strong } \\
\text { participation, the social media can still make the users feel its authenticity. So the information spread on the } \\
\text { social media is more convincible. }\end{array}$
\end{tabular}

\section{The troubles between the consumers and the enterprises face under the traditional Internet marketing mode.}

The traditional marketing model, represented by the viral marketing, is based on the user's public praise on the internet, Spreads and diffuses the information like a virus, which makes use of rapid replication to convey the information to the audience. The viral marketing uses an Infected attractive "pathogen", through spreading the "pathogen" once again , like a virus, its quantity will make a geometric progression increase. In the early period of the viral marketing occurrence, because of its low cost, high transmission ability, the characteristic to attract the users' eyeballs, it rises rapidly, and makes the emergence of a large number of virus marketing activity cases, such as " to eat the Pizza Hut down", and the Gmail promotion activities.

The most famous one is the case of "Ban " wang lao ji in 2008 . After the earthquake broke out in Wenchuan, many national 
ministries and the CCTV jointly organized a fundraising party on the night of May 18th. On behalf of the JDB group (Wang Laoji: The Chinese old trademark, currently is used by three enterprises in common, including the Wang Laoji in Guangzhou in charge of the mainland market, Wang Laoji International in Hongkong in charge of the market of Hongkong and the overseas market, and the JDB limited company(Guangzhou) who has leased the right to use the trademark for 20 years) , Mr. Yang donated I billion yuan, the domestic single donation maximum, which made Wang Laoji become the focus of attention. On May 19th, in the domestic Internet community, the posts are so popular known as "ban Wang Laoji" ,in light of the good deeds by Wang Laoji , the internet users were called for to clear the Wang Laoji cool tea in the supermarkets, within 3 hours, the posts about Wang Laoji were posted over 140,000. Later, the news about which the Wang laoji were "sold out" in some regions was reported again and again . Almost overnight the southern cool tea "Wang Laoji" became famous all over the country, some people began to write "Drink the canned Wang Laoji." as their MSN signature. the prevalence of viral marketing, has brought the huge impact to the network information dissemination and the Internet users, making the Internet information specifications a great focused issue.

On the one hand, because the viral marketing uses exaggeration, luminous language and styles to attract the attention of network users, it makes up a powerful public opinion, making the information spread so largely and rapidly that covers the normal and rational information, and breaks the original information dissemination mechanism, destroys the normal route of transmission of the information. On the other hand, the powerful network public opinion leads to the occurrence of the irrational events.

In the "Yao Jiaxin " case, the justice anxiety of the citizens makes the case become a public event, where appears the "spiral of silence Process "(a public opinion learning concept, is refers to the invisible pressure to the minority's opinion due to the existing majority public opinion, it forms a spiral process where a public outcry is from one side, while ,the other party is more silence, so a more powerful public opinion comes into being), which is the result of the group psychological effects.

In addition, the viral marketing has produced a controversial industry -the Internet ghostwriters. the Internet ghostwriters are the network personnel who are employed by the network public relations companies, to put forward or reply to the posts to support their opinions. In the promotion ,they play a positive role,But in some mass incidents, through the malicious attacks, the Internet ghostwriters disrupt the order of the Internet, reducing the "gatekeeper" function of the media industry, and doing harm to the trust of the users for network media and network information.

The bigotry, the violence, and irrational behavior of the network citizens aroused by the network ghostwriters have been concerned by the whole society. During the two national sessions In 2011, some representatives pointed out: "the network ghostwriters, mainly represented by the public relationship companies, are the network "evil forces" who help others post, delete, support their posts to make a public opinion trend, only seeking their evil interest, which destroys the network ecosystem, leading to vicious competition, misleading the public opinion, becoming the troublemakers which overthrow a well-ordered morality, must be band at once on the legislative level. "

In 2010 March , A project survey (2359 people participated ) on the public opinion network and Sohu network was carried out by the China youth daily social survey center , $90.3 \%$ of respondents worry about that more and more network hype will lead to the public trust crisis of network information. For the hyped, and popular events or characters in the network, only $2.1 \%$ netizens think they are real.

Deterioration of the network environment, the lack of ethics and trust, bring negative effects to consumers' choices in shopping and the reputation trust, reduces the effects of the traditional network marketing, under the network marketing mode, the traditional consumers and companies are facing a dilemma. 


\section{Consumers' confusion when face choices}

People are rational, as Adam Smith, Thomas Malthus and other economists thought. Although the criticism of the hypothesis of rational man in the academic world has never been stopped, such as Maslow's demand theory, Simon's bounded rationality theory, and the complex, and multi-faces of humanity elaborated by Douglas c. North in his "The system, Institutional change and economic performance ". but what is undeniable is that people are still rational. In "Das Kapital" , Marx said: "in the analysis of the economic form, we cannot use neither the microscope nor the chemical reagent, instead, we must use the abstraction power."

The traditional Internet marketing mode relies mainly on the celebrities endorsement or the "virus" type information dissemination to influence the consumers. The former is to transplant the television ads, the print ads, and other forms of advertisings to the Internet, while, the latter is to construct the powerful pressure of public opinion to influence the consumers' decision. Both modes are traditional marketing, through the stars or the opinions' Whirlpool, forcing the information into the consumers' minds, which does not accord with the modern marketing concept, and also violate the cognition laws of the consumers. Facing the huge and rapid flows of information transformation, consumers are involved in two confusions : Firstly, the authenticity is doubted, whether the information is true or not ,the anxiety of which is aroused by the excess information ; Secondly ,the confusion about the suitability of the information, which is from the rejection bandwagon effects, is about whether the information is proper for their questions.

The culprit for both the consumers' confusions are mainly from three aspects: the richness of information , the emergence of the hierarchical network structure, and the occurrence of fraud online.

\section{The enterprises' marketing dilemma}

(1) The location dilemma

Although there are many successful network marketing cases, the enterprises still have some confusions and doubts when facing the network marketing, most of whom still doubt the effects of the network marketing.

(2) The users' reliability dilemma

With the advantages of low cost, fast transmission ability, the network marketing were also criticized for its bad style, short residence time, and its difficulties on weakened viscosity, some enterprises tend to carry on the network marketing, but do not know how to proceed it.

(3)the correspondence between the consumers' confusions and the enterprises' plights

The consumers doubt the accuracy of the information, and whether the information is suitable for their needs, while the dilemmas of the enterprises' performances are whether their service is matching the consumers' needs and the adhesiveness of their consumers. But there is certain conjunction between the sides.

\section{The construction of social media marketing mode}

As we have discussed, the traditional network marketing is limited by the weakness of the network marketing, it cannot build an effective connection to the consumers, to maintain their consumer consistency, which makes them into a dilemma, where on the one hand, the enterprises are eager to use the network marketing for its advantages of its low cost, and fast transferring speed, etc; on the other hand ,they are afraid of it for so many fail cases in the enterprises' network marketing effects. The plight will be solved only through the new social media marketing, in fact, it has been proved the social media marketing, really expresses its advantages of network marketing - the accuracy of targeting and its rapid spread speed.

\section{1 .Under the perspective of social network , the group consistence and the group trust of the social media.}

The social media expand our social network greatly. We can consistently concern others and be concerned online, through all kinds of relationships .But why the social media marketing can overcome the consumers' confusions and the enterprises' dilemmas of the traditional network marketing, fundamentally 
lies in the social network under the social media, which is based on the real network made up of the friends in reality, and can make the users have the group consistence and eliminate the confusions brought by the internet.

\section{The integrate of the consumers and the enterprises under the perspective of the social network}

(1) The enterprises integrate themselves into the consumers' social network

The enterprises integrate themselves into the consumers' social network, firstly through the word-of-mouth, which is the oral spreading from one to another among people. It is essentially the process of the dissemination of the information , the subject of which is the consumers, the contents of which are the consumers' experiences, the satisfaction and the personal enterprise's image, and the target of which is the social network related group. Through the dissemination of the information, the enterprises have more knowledge and cognition of their users.

The word-of -mouth is spread mainly through the way of the individual social network . the accesses are mainly through the individual weak or strong relations. The enterprises integrated into the consumers' network through the word-of mouth through mainly the following 2 processes:

Firstly, one important access of the word-of-mouth is the weak relation. Limited by the individual energy and time restriction, the strong relations mastered by people have limitation. In the social relations, to get the maximum social resources, we must frequently make use of the weak relations. Which makes it own the transmissibility. For the information, the weak relation also owns the important transmissibility. For the existence of people' psycho curiosity , the information heard by accidence from the weak relations usually can be spread by the individuals. So, Granovetter said "Although not all the weak relations can be served as the information bridge, but what is served as the information bridge is only the weak relation."

Suppose that a enterprise's word-of mouth hasn't arrived at the social network branch, where the users have no cognition of the enterprise. So, the word-of-mouth can access only by the weak relation.

Secondly, after the word-of-mouths get into the social network branch through the weak relation, it will be spread effectively through the strong relation. The strength brought by the strong relation secures the effects of the word-of-mouth, having effects on the consumers' decisions of their behaviors. Just because the traditional network marketing cannot get into the consumers' strong relations, its shortcoming for the weakness of transmissibility is caused. Through the strong relations, the consumers can pass the information, and their opinions and viewpoints to others, as well as having affects on them.

Because the connections happen frequently in the strong relations, the word-of-mouth is not something which happens by accident, but a stable and consistent spreading. In reality, some consumers would like to promote some positive word-of-mouths actively. This promotion will pass the information to the people around repeatedly within a pretty long time. For example, we may meet some situations like this ;When a friend had an amazing experience of some product, he will pass his experience to the surroundings consistently within the following short time, there will always be a phenomenon - - even he is tired of narrating it, he still continue doing so. So the strong relations deepen people's impressions, and drop a hint on their psycho. When they see the same product, they will connect it to the consumer's experience quickly. As a result, the effects of the word-of -mouth is fulfilled.

Making use of the weak or strong relations, the enterprises propagate themselves with maximize promotion, and will be known and learned by the consumers.

(2) The trust constructed between the consumers and the enterprises

The social media makes the trust constructed between the consumers and the enterprises ; on the one hand the consumers trust the enterprises, on the other hand, the enterprises trust the consumers.

Firstly, how the social media make the consumers trust the enterprises. After the enterprises enter the consumers' social 
network through the weak and strong relations, which doesn't mean the trust can be formed spontaneously. The trust cannot be passed simply. That is, when A trusts B, B trusts C, A won't trust $C$ definitely, especially when the enterprises enter the strong relation social network, the consumers' doubts can't be avoided. But the social media has the advantage to help bring about the construction of trust between the consumers and the enterprises.

The social media has undefined communication functions, its view functions help us know the others' social network, and find the valuable social resources. Specifically, through the "attentions" and "fans" of our friends, we know his contacts, so that we have found that the friends can provide us what kind of social relations. This function can offer the maximize value for communication, expand the individual's Interpersonal relationship, and also meet the needs of the individuals.

Secondly, the trust of the enterprises will respond to the trust of the consumers. The social media marketing idea is to respect the consumers' self-determination, they believe the consumers know their own needs, the production of the enterprises can't break away from the markets, to design , manufacture, and promote their products. But it should integrate itself with the consumers' wisdoms, through their own experiences, to refine a better idea, and satisfy the potential needs of the consumers.

\section{The measures carried out by the social media marketing}

The measures carried out by the social media marketing mainly include : the involvement of the social media, the focus on the users' concerns , and the "conversation" style of communication with their users.

\section{1 .The involvement of the social media}

To adopt the social media marketing, and form a stable relationship with the users on social network, the enterprises are demanded to get access to their users initiatively and actively involve themselves in the social media.

\section{2 .Focus on the users' concerns}

To create the excellent contents, the following aspects should be concerned :

Firstly, focus on the users, the excellent contents can be realized their valued to the enterprises only by being accepted and understood by the users. So the enterprises must know the values and needs of their users, and create their contents based on the users' needs, which is also the basis of the social media marketing. The process for the enterprises to know their users through the social media is not only simple, but also complicated. On the one hand, the consumers' needs will be expressed under the social media ,through the network , shared on the friends net and their social relations' circles which makes it easy for the enterprises to know their users. On the other hand, in the consistent communications and shares, the users' needs become more complex and harsh, the unsatisfied needs will cause the bad moods of the users , whose spreading in the group will bring about the negative effects to the enterprises. So the enterprises must concern about the related topic on their businesses, know their users' new ideas, perceive the possible negative information ,all of this can help provide evidences to them to adopt the prompt and effective measures.

Secondly, Self-awareness. The excellent contents contain the information of

The enterprise's strategies and its culture. Integrating its strategies and its culture with the contents is the only way to make them into the users' cognitions. So the enterprise must be aware of its own strategies and its own culture, and make its contents serve for them.

Thirdly, the language style should be adaptive to the users. Under the internet, the language style should be free and creative. The enterprise carrying out the social network marketing should avoid the expressions through the blunt terms and decrease using internal terms used in the enterprise's symposiums, which will increase the users' sense of distance. The enterprise should follow the users' language habits. The expressions and words used in the internet are consistently redefined, enriched and enlarged greatly. Based on the knowledge of the users, the enterprise should choose 
the adaptive language style, neither exaggerate nor out-of-date.

Fourthly, spread the contents widely. The enterprise creates the contents and passes them to the users through various accesses under the social media, the accesses to convey the information are increased. Besides the e-mail ,blogs, and forums, the social media also makes use of the RRS, "Really Simple Syndication", and the dissemination between the users' concerns ,etc. to make good use of the RSS and the users' concerns, the enterprise spreads information on the excellent RSS sources or the highly concerned users, the contents are spread in the way of chain propagation in the social network, the spreading range of which are expanded.

\section{3 .Build up constantly the conversation style marketing}

Conversation is the best way to construct connections between the enterprise and its users. Persistent conversations can bring the distance closer, and help them find where their contradictions lie. The enterprise can not only spread its contents on the social media, but also make its users involved in the communications, and respond to them actively. The social media eliminates the feelings of distance, and hierarchy. So everyone can speak to the others as he likes. Such as using the function @ sina blogs, where the common users use the function to @ certain stars, and talk to their idols directly. That expresses the free, equal social network structure. In a word, the enterprises should abandon the holy positions, and talk to their users actively.

\section{Conclusion}

Social media is a result of the development of the Internet. In the web2.0 era, the Internet applications advocate the User-generated content (UGC), So appeared the search engines, blogs, etc. Although these applications enlarge the users' network expansion, and make it not be limited by time and space to share their contents. While, Its essence is still "a Point to the group ", that is, the connection between the user and the platform . Due to the weakness of individual voice, the user must submit to the point of view of the mass, and the mainstream . Therefore, this link will lead to stratification of the network users in the practical communication. The celebrity blogs, the community site moderators become the top class, while, a large number of users become the lower class. The appearance of network classes is a breach of the Internet nature of equality, fairness, which ignores the main body of the network - the wide "grass root "users. Therefore, as the development of the Internet, the social media, which takes weibo, social networking sites as its representatives, is bound to come into being as a more equal and free platform.

\section{Reference:}

[1]. "The analysis of the social network" by Fei Zhonglin, and Wang Jingan : a managing study method and perspective. The science managing study [J], 2010 issue 24 .

[2]. "The connotation, extension, reengineering of the enterprise marketing modes " by Xu Qianli. Business Times [J],2008 issue 24 .

[3]. "The overview of the consumers' behaviors study under the social network marketing mode " by Lu Wenjuan, Han Dechang Dynamic economics[J],2010, issue 7.

[4]. "The analysis of eight major network marketing modes" by Shu-dong zhou,, Business Research [J], 2008. (22) : 177-179

[5]. "The development and innovation of network models" by Jian, Chen, and Kua-Yao chang , [J] Commercial Age, 2008. (52) : 83-84

[6]. "Research of Web 2.0 network marketing model" by Yao Zhongjie [D] Shanghai: The east China normal university, in May 2007.

[7]. "The research on Chinese enterprise network marketing model and the performance evaluation " by Wang Yan [D] Harbin: Harbin institute of technology, May 2006

[8]. " The Study of the patterns of Chinese enterprise network marketing" by Zhang Jinan [D]: Shandong university, March 2010

[9]. "The research on the role of users' experience in the search engine marketing strategies " by Xiao-li He [D] Jinan: Shandong university, March 2010 